\title{
Optimizing low-order controllers for haptic systems under delayed feedback ${ }^{2 / 5}$
}

\author{
Bogdan Liacu ${ }^{\mathrm{a}, \mathrm{c}}$, Ahmet Taha Koru $^{\mathrm{b}}$, Hitay Özbay ${ }^{\mathrm{b}}$, Silviu-Iulian Niculescu ${ }^{\mathrm{a}, *}$, Claude Andriot $^{\mathrm{c}}$ \\ ${ }^{a}$ Laboratoire des Signaux et Systèmes (L2S), CNRS-SUPELEC, 3 rue Joliot Curie, 91192 Gif-sur-Yvette, France \\ b Department of Electrical \& Electronics Engineering, Bilkent University, Ankara, Turkey \\ ' CEA, LIST, Interactive Robotics Laboratory, Fontenay aux Roses, F-92265, France
}

\section{A R T I C L E I N F O}

\section{Article history:}

Received 20 February 2012

Accepted 1 January 2013

Available online 1 March 2013

Keywords:

Haptics

Teleoperation

Time-delay

H-infinity optimization

Stability limits

PID control

\begin{abstract}
A B S T R A C T
In this paper, a PD controller design for haptic systems under delayed feedback is considered. More precisely, a complete stability analysis of a haptic system where local dynamics are described by some second-order mechanical dynamics is presented. Next, using two optimization techniques $\left(H_{\infty}\right.$ and stability, margin optimization) an optimal choice for the controller gains is proposed. The derived results are tested on a three degree-of-freedom real-time experimental platform to illustrate the theoretical results.
\end{abstract}

(c) 2013 Elsevier Ltd. All rights reserved.

\section{Introduction}

During the last decade, virtual environments have become very popular and are largely used in many domains as, for example, prototyping (see, for instance, Fig. 1(a) for an appropriate example of prototyping using haptic interfaces and virtual environment Sreng, Lécuyer, Mégard, \& Andriot, 2006), training for different devices and assistance in completing difficult tasks (see Fig. 1(b) for some virtual environment used for task assistance/supervision David, Measson, Bidard, Rotinat-Libersa, \& Russotto, 2007; Gosselin et al., 2010).

Understanding the interaction between humans and robots is at the origin of developing several control schemes for teleoperation systems. Roughly speaking, teleoperation extends, at some level, the human capacity in manipulating objects remotely by providing the corresponding operator with similar conditions as those encountered at the remote location (see, for instance, the surveys by Hokayem \& Spong, 2006; Sheridan, 1993). Among the recent applications, one may cite telesurgery and space telerobotics (see, e.g., Aziminejad, Tavakoli, Patel, \& Moallem, 2008 and the references therein), both involving long distance

\footnotetext{
Some of the results proposed in this paper have been partially presented at the 10th IFAC Workshop on Time-Delay Systems (TDS), Boston, USA under the title: Low order controller design for haptic systems under delayed feedback.

* Corresponding author. Tel.: +33169851711, fax: +33169851765

E-mail addresses: bogdan.liacu@supelec.fr (B. Liacu),

koru@ee.bilkent.edu.tr (A. Taha Koru), hitay@ee.bilkent.edu.tr (H. Özbay), Silviu.Niculescu@Iss.supelec.fr (S.-I. Niculescu), claude.andriot@cea.fr (C. Andriot).
}

communication between master and slave devices. Furthermore, in both cases, haptic feedback proved its potential in improving corresponding task performance. In this context, time-delays appear as natural components of the closed-loop schemes in order to describe some of the dynamics induced by the communication channels with strong impact on (asymptotic) stability and transparency (see, e.g., Gil, Sanchez, Hulin, Preusche \& Hirzinger, 2007). ${ }^{1}$ It is worth mentioning that, in haptic systems, excepting the communication channel, time-delays may appear also as intrinsic components of the processing time for the virtual reality environment. Indeed, in free motion, the delay effect can be felt by the viscosity phenomenon (high force feedback felt at the haptic interface end) and such a property is completely lost in the case of a "hard"-contact with the environment.

In the open literature, there exists several control methods used in teleoperation and further adapted for haptics. In this sense, the following methods are mentioned: ProportionalDerivative (PD) with local dissipation (Lee \& Spong, 2006), PD with passivity observer (Artigas, Vilanova, Preusche, \& Hirzinger, 2006; Ryu, Kwon, \& Hannaford, 2002a,b), PD with passive setpoint modulation (Lee \& Huang, 2008), wave scattering transform (Niemeyer, 1996; Niemeyer \& Slotine, 2004) and Smith predictor (Cheong, Niculescu, \& Kim, 2009). Comparative studies of these methods in the case of teleoperation systems as well as of haptic

\footnotetext{
${ }^{1}$ By transparency is understood the capability as well as the impression of operating directly on a remote environment independently of the presence of master and slave units (Lawrence, 1993; Yokokohji \& Yoshikawa, 1994).
} 

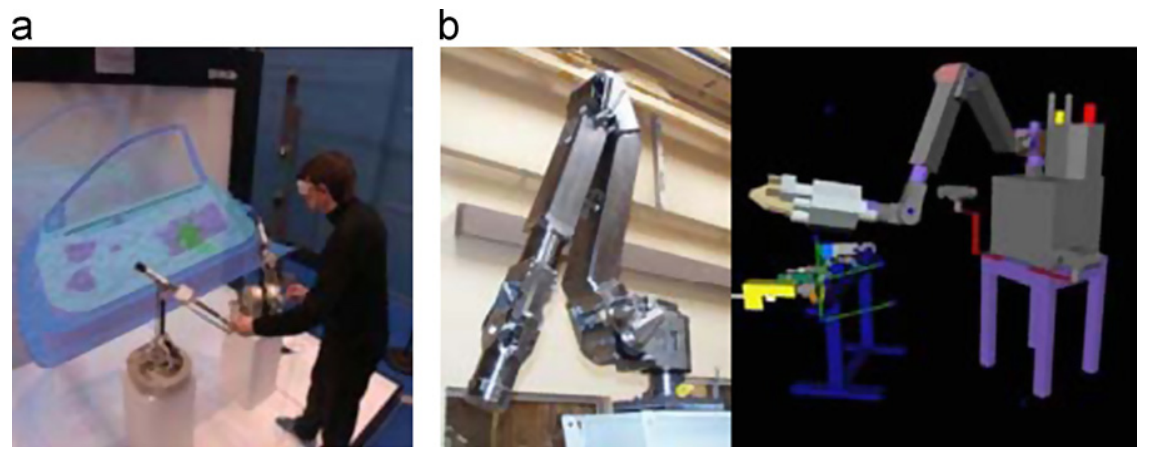

Fig. 1. Examples of virtual environments applications. (a) Virtual prototyping. (b) Virtual assistance/supervision.

systems can be found in the literature, as for example, (Rodriguez-Seda, Lee, \& Spong, 2009) or (Liacu et al., 2012; Sankaranarayanan \& Hannaford, 2008), respectively. For instance, Rodriguez-Seda et al. (2009) compares existing algorithms for motion and force control of some bilateral teleoperation schemes with a particular attention paid to Internet ${ }^{\mathbb{R}}$-based teleoperation. Next, Sankaranarayanan and Hannaford (2008) focuses on the performances analysis of a peer-to-peer haptic collaborative system including two users manipulating same object simultaneously. Finally, Liacu et al. (2012) presents a comparative study of some of existing control architectures for haptic systems subject to communication delays.

In the sequel, the closed-loop stability analysis of some class of practical bilateral haptic systems coupled with a virtual environment by using a standard proportional-derivative (PD) control law is addressed. The time-delays in the communication channels are assumed to be constant and, as it will be seen, only the overall time-delay (the sum of the forward and backward timedelays) needs to be known. There exists an abundant literature on PID control for time-delay systems (see for instance, O'Dwyer, 2000; Silva, Datta, \& Bhattacharrya, 2005 and the references therein) and most of the existing methods are computationally involved.

The methods proposed in the paper are original, in our opinion, and they exploit the particular structure of the closed-loop quasipolynomials. The derived stability conditions are necessary and sufficient and, to the best of the authors' knowledge, such a characterization is new. Furthermore it allows a simple construction of the corresponding stability regions in the controller parameterspace. Next, as a byproduct of the analysis, the computation of the optimal controller gains by using two particular frequency-domain techniques $\left(H_{\infty}\right.$-based design and fragility ${ }^{2}$ analysis $)$ is proposed. To the best of the authors' knowledge, the optimization of the controllers' gains represents a novelty making the contribution original. Finally, the derived control law are validated on some illustrative example involving a virtual spherical mass moving in an appropriate $3 \mathrm{D}$ virtual scene and the study is performed by considering a complete scenario from free to some restricted motions.

The remaining paper is organized as follows: in Section 2, a general haptic system scheme including communication channels is introduced. Next, Section 3 is devoted to the stability analysis in closed-loop in the presence of PD or PD-like control laws. In particular, the approach proposed allows recovering a stability condition derived in Nuno, Ortega, Barabanov, and Basanez (2008) by using a different methodology. Section 4 focuses on

\footnotetext{
${ }^{2}$ Here, by fragility, it is simply understood the deterioration of closed-loop stability due to small variations of the controller parameters (see, for instance, Alfaro, 2007; Keel \& Bhattacharyya, 1997; Makila, Keel, \& Bhattacharyya, 1998 for further details on such topics).
}

an appropriate optimal choice for the controller parameters by using the (frequency-domain) approaches mentioned above. The experimental validation of the proposed methodology is discussed in Section 5 on a simple three degree of freedom haptic system. Finally, some concluding remarks end the paper.

\section{System description}

In Fig. 2, a general scheme of a haptic system is presented. The ideal haptic system should satisfy simultaneously the following conditions:

- first, the position tracking error has to be as small as possible between the haptic interface and the virtual object,

- second, the system has to have a high degree of transparency, i.e. in the "free" motion case, the force feedback felt at the haptic interface end must be as small as possible and in the case of a "hard"-contact, a stiff response is desired.

Next, Fig. 3 presents the general control scheme of a haptic interface and a virtual environment including control feedback.

The starting point is represented by the classical dynamic (nonlinear) equations of motion for two robots in the haptics framework. More precisely, the corresponding dynamics write as

$M_{h}\left(x_{1}\right) \ddot{x}_{1}(t)+C_{1}\left(x_{1}, \dot{x}_{1}\right) \dot{x}_{1}=-F_{1}(t)+F_{h}(t)$,

$M_{v}\left(x_{2}\right) \ddot{x}_{2}(t)+C_{2}\left(x_{2}, \dot{x}_{2}\right) \dot{x}_{2}=F_{2}(t)-F_{e}(t)$,

where $x_{1}, x_{2}$ are the haptic interface/virtual object position, $F_{h}, F_{e}$ are the human/environmental forces, $F_{1}, F_{2}$ are the force control signals, $M_{h}, M_{v}$ are the symmetric and positive-definite inertia matrices, and $C_{1}, C_{2}$ are the Coriolis matrices of the haptic interface and virtual object systems, respectively. The central idea of the control scheme is to use two similar PD controllers, one for controlling the haptic interface and another for the (corresponding) virtual object. In such a configuration, the controllers' equations are then given as follows:

$$
\begin{aligned}
& F_{1}(t)=\underbrace{K_{d_{1}}\left(\dot{x}_{1}(t)-\dot{x}_{2}\left(t-\tau_{2}\right)\right)+K_{p_{1}}\left(x_{1}(t)-x_{2}\left(t-\tau_{2}\right)\right)}_{\text {delayed D-action }}, \\
& F_{2}(t)=\underbrace{K_{d_{2}}\left(-\dot{x}_{2}(t)+\dot{x}_{1}\left(t-\tau_{1}\right)\right)+K_{p_{2}}\left(-x_{2}(t)+x_{1}\left(t-\tau_{1}\right)\right)}_{\text {delayed P-action }},
\end{aligned}
$$

where $\tau_{1}, \tau_{2}$ are the forward and backward finite constant timedelays and $K_{p_{1}}, K_{d_{1}}, K_{p_{2}}, K_{d_{2}}$ are the PD control gains corresponding to the haptic and virtual controller respectively, see Fig. 4. 


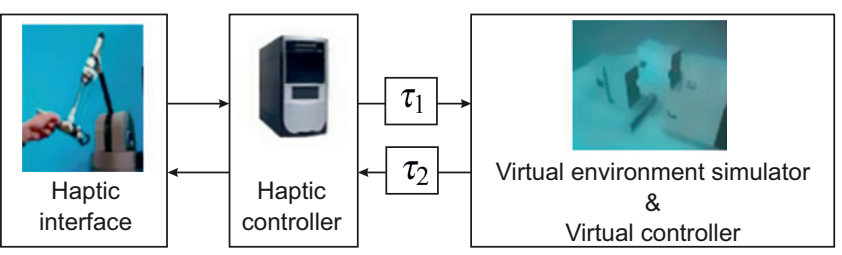

Fig. 2. General scheme of a haptic system.

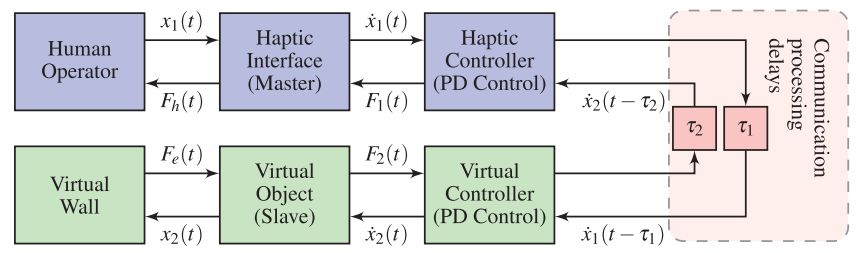

Fig. 3. General PD control scheme for haptic systems.

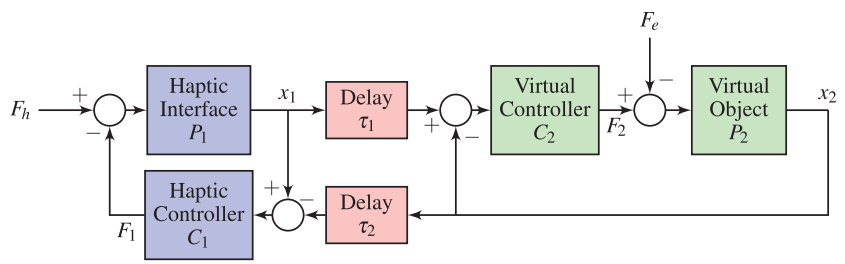

Fig. 4. Bilateral haptic system.

\section{Stability analysis}

\subsection{PD control}

From Fig. 4, the equations describing the system response can be written as follows:

$X_{1}(s)=P_{1}(s)\left(F_{h}(s)-C_{1}(s)\left(X_{1}(s)-e^{-\tau_{2} s} X_{2}(s)\right)\right)$,

$X_{2}(s)=P_{2}(s)\left(-F_{e}(s)+C_{2}(s)\left(-X_{2}(s)+e^{-\tau_{1} s} X_{1}(s)\right)\right)$,

where $X_{i}(s)$ denotes the Laplace transform of the time signal $x_{i}(t)$, $i=1,2$; similarly for $F_{h}(s)$ and $F_{e}(s)$; here, $\tau_{1}>0$ and $\tau_{2}>0$ denote the corresponding (forward and backward) time-delays. Transfer functions $P_{i}(s)$ and $C_{i}(s)$ are taken as follows (position available for measurement and PD structure for the control law):

$P_{1}(s)=P_{2}(s)=\frac{1}{s(m s+b)}=: P(s)$,

$K_{p_{1}}=K_{p_{2}}=: K_{p}, \quad K_{d_{1}}=K_{d_{2}}=: K_{d}$,

$C_{1}(s)=C_{2}(s)=K_{p}+K_{d} s=: C(s)$.

It is worth mentioning that the robots are modeled as linear systems since the haptic interface does not present any particular behaviors that are not covered by the linear model, and the virtual robot is represented by an ideal case.

As far as the internal stability analysis is concerned, the above system is equivalent to a system where the controller is of PI type (of the form $K_{d}+K_{p} / s$ ), and the process (measured) variable is represented by the velocity, i.e., process given by: $(m s+b)^{-1}$.

By rearranging (5) and (6) above, one obtains:

$$
\begin{gathered}
{\left[\begin{array}{cc}
1+P_{1}(s) C_{1}(s) & -P_{1}(s) C_{1}(s) e^{-\tau_{2} s} \\
-P_{2}(s) C_{2}(s) e^{-\tau_{1} s} & 1+P_{2}(s) C_{2}(s)
\end{array}\right]\left[\begin{array}{l}
X_{1}(s) \\
X_{2}(s)
\end{array}\right]} \\
=\left[\begin{array}{c}
P_{1}(s) F_{h}(s) \\
-P_{2}(s) F_{e}(s)
\end{array}\right] .
\end{gathered}
$$

Therefore, with the process (plant) and controller definitions (7)-(9), the characteristic equation of the feedback system in closed-loop can be written as follows:

$(1+P(s) C(s))^{2}-(P(s) C(s))^{2} e^{-\left(\tau_{1}+\tau_{2}\right) s}=0$,

which is simply equivalent to

$\chi_{1}(s) \chi_{2}(s)=0$,

where

$\chi_{1}(s):=\left(1+P(s) C(s)+P(s) C(s) e^{-\tau s}\right)$,

$\chi_{2}(s)=:\left(1+P(s) C(s)-P(s) C(s) e^{-\tau s}\right)$,

and $\tau:=\left(\tau_{1}+\tau_{2}\right) / 2$.

Remark 1. An analysis of equations of the form (12) has been given in Shayer and Campbell (2000) for some particular class of first-order quasipolynomials encountered in neural network models, without any attempt to consider the general case. Different approaches for the closed-loop stability analysis can be found in Morarescu, Mendez-Barrios, Niculescu and Gu (2011), Liacu, Mendez-Barrios, Niculescu, and Olaru (2010), Saeki (2007), Michiels and Niculescu (2007) and the references therein. In this paper, a different analytical approach is considered. Such an approach makes use of the gain and phase margins estimation in order to perform the stability analysis of such a feedback system.

The following result is obtained (see Appendix A for the proof)

Theorem 1. The bilateral haptic system is asymptotically stable independent of the delay values $\left(\tau_{1}, \tau_{2}\right)$ if the controller gains satisfy the condition

$K_{d} \geq \frac{m}{b} K_{p}$

Furthermore, when $K_{d} / K_{p}<m / b$, there exists two cases:

(a) If $0<m K_{p}-b K_{d}<b^{2} / 2$, then the feedback system is stable independent of the delay values $\left(\tau_{1}, \tau_{2}\right)$.

(b) If $m K_{p}-b K_{d}>b^{2} / 2$, then the closed-loop system is stable if and only if

$m K_{p}-b K_{d}<\frac{b^{2}}{2}\left(1+\omega_{0}^{2}\right)$

where $\omega_{0}>0$ is the solution of the equation:

$$
\frac{\pi-2\left(\tan ^{-1}(x)-\tan ^{-1}\left(\frac{b K_{d}}{m K_{p}} x\right)\right)}{x}=\frac{\left(\tau_{1}+\tau_{2}\right) b}{2 m} .
$$

From the conditions of Theorem 1, the allowable range of $m K_{p} / b^{2}$ and $K_{d} / b$ for all $b / m>0$ can be explicitly determined. The corresponding stability region is shown for three different timedelay values in Fig. 5 (and for some different large time-delay values in Fig. 6).

\subsection{PD-like control}

In Nuno et al. (2008), the authors proposed a PD-like controller, having the block scheme presented in Fig. 7.

More precisely, only the position error will be used in order to guarantee the passivity of the system. With this assumption, Eqs. (3) and (4) are rewritten as follows:

$F_{1}(t)=\underbrace{K_{d_{1}} \dot{x}_{1}(t)}_{\text {D-action }}+K_{p_{1}}\left(x_{1}(t)-x_{2}\left(t-\tau_{2}\right)\right)$, 


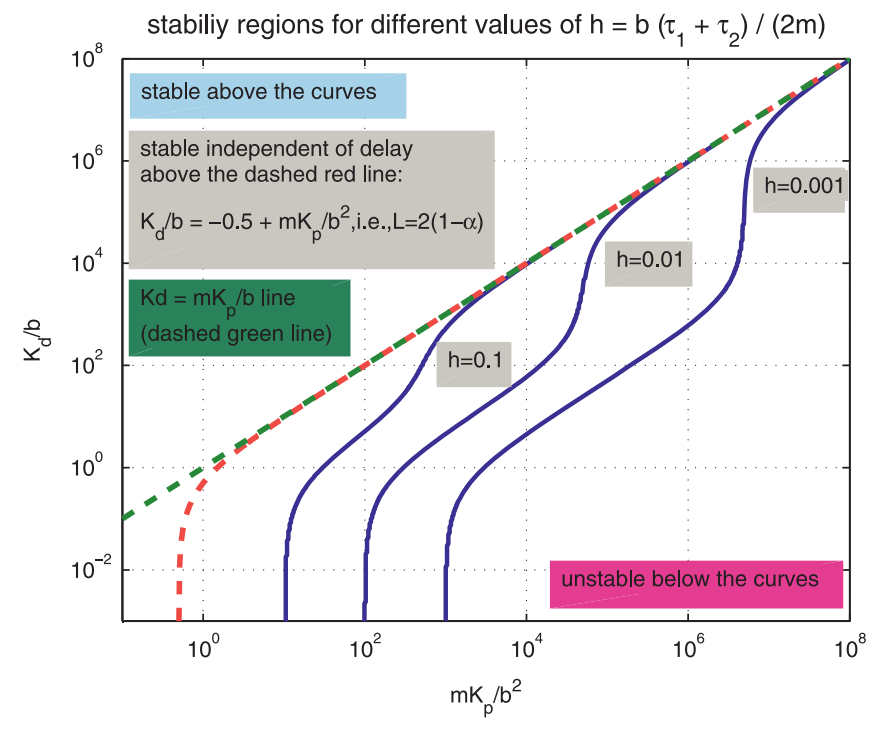

Fig. 5. Allowable region of controller parameters for stability of the bilateral haptic system.

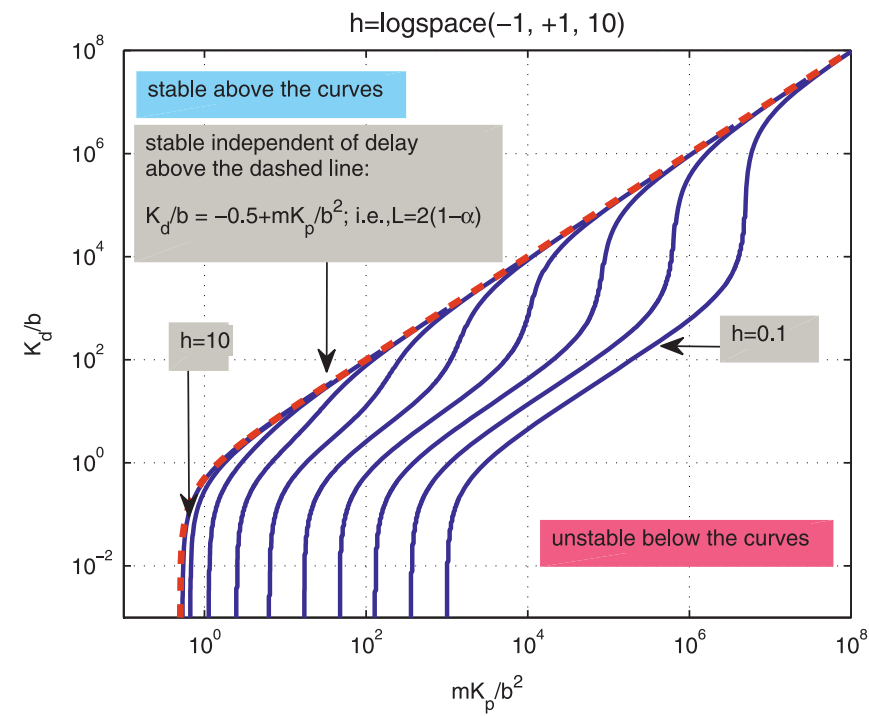

Fig. 6. Allowable region of controller parameters for stability of the bilateral haptic system.

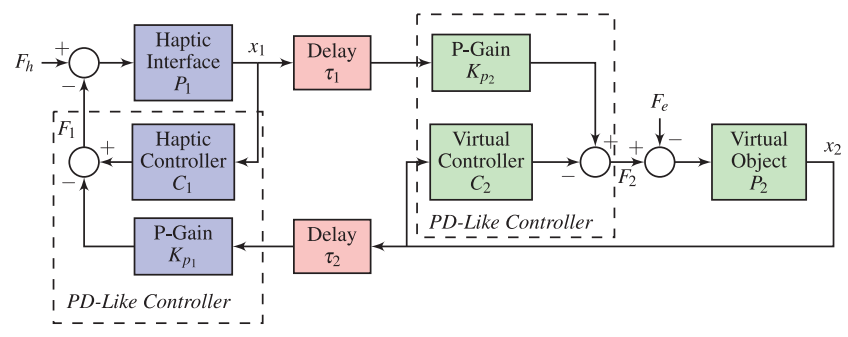

Fig. 7. Bilateral haptic system using a PD-like controller.

$F_{2}(t)=\underbrace{-K_{d_{2}} \dot{x}_{2}(t)+K_{p_{1}}\left(-x_{2}(t)+x_{1}\left(t-\tau_{1}\right)\right)}_{\text {D-action }}$,

Next, by considering (7)-(9), Eqs. (5) and (6) become

$X_{1}(s)=P_{1}(s)\left(F_{h}(s)-C_{1}(s) X_{1}(s)-K_{p_{1}} e^{-\tau_{2} s} X_{2}(s)\right)$,

$X_{2}(s)=P_{2}(s)\left(-F_{e}(s)-C_{2}(s) X_{2}(s)+K_{p_{2}} e^{-\tau_{1} s} X_{1}(s)\right)$.
Rearranging (18) and (19), it follows:

$$
\begin{gathered}
{\left[\begin{array}{cc}
1+P_{1}(s) C_{1}(s) & -K_{p_{1}} P_{1}(s) e^{-\tau_{2} s} \\
-P_{2}(s) K_{p_{2}} e^{-\tau_{1} s} & 1+P_{2}(s) C_{2}(s)
\end{array}\right]\left[\begin{array}{l}
X_{1}(s) \\
X_{2}(s)
\end{array}\right]} \\
=\left[\begin{array}{c}
P_{1}(s) F_{h}(s) \\
-P_{2}(s) F_{e}(s)
\end{array}\right] .
\end{gathered}
$$

Therefore, with the definitions (7)-(9), the new characteristic equation of the feedback system becomes

$(1+P(s) C(s))^{2}-K_{p}^{2} P(s)^{2} e^{-\left(\tau_{1}+\tau_{2}\right) s}=0$,

which is equivalent to

$1+P(s) C(s) \pm K_{p} P(s) e^{-\tau s}=1 \quad+K_{p} s P(s)\left(\frac{K_{d}}{K_{p}}+\frac{1}{s} \pm \frac{e^{-\tau s}}{s}\right)=0$.

Since $\left(K_{p} S P(s)\right)$ is positive real, in order to guarantee the stability, it is needed to ensure that

$\operatorname{Re}\left(\frac{K_{d}}{K_{p}}+\frac{1 \pm e^{-\tau s}}{s}\right)>0 \quad \forall s \in \mathbb{C}_{+}$.

Knowing that

$\operatorname{Re}\left\{\frac{K_{d}}{K_{p}}+\frac{1+e^{-j \tau \omega}}{j \omega}\right\}=\frac{K_{d}}{K_{p}}-\frac{\sin (\tau \omega)}{\omega} \geq \frac{K_{d}}{K_{p}}-\tau$

and

$\left|\frac{1-e^{-\tau j \omega}}{j \omega}\right| \leq \tau \quad \forall \omega \in \mathbb{R}_{+}$,

the stability is guaranteed if the following condition holds:

$\frac{K_{d}}{K_{p}}>\tau \Leftrightarrow K_{d}>K_{p} \tau$.

The result obtained in Nuno et al. (2008), by using a different argument

$K_{d_{1}} K_{d_{2}}>K_{p_{1}} K_{p_{2}} \tau_{1} \tau_{2}$

is exactly the same with (23), under the assumption (8) and $\tau_{1}=\tau_{2}=\tau$.

\section{Optimal gains}

In this section, optimal gains $K_{p}$ and $K_{d}\left(H_{\infty}\right.$-base, non-fragility) are presented and discussed, for the PD control configuration studied in Section 3.1.

\section{1. $H_{\infty}$-based design}

Let us define the position tracking error

$e(t):=x_{1}(t)-x_{2}(t)$.

From (10), it is computed

$E(s)=\frac{P(s)}{1+P(s) C(s)+P(s) C(s) e^{-\tau s}}\left(F_{h}(s)+F_{e}(s)\right)$.

While trying to make the error small, one may be forced to use "high" command signals which may lead to actuator saturation. Since large control signals are not desirable, it is also wanted to "penalize" the control. Again, from (10), the output of the controller, $F_{2}(t)$, on the virtual side can be computed as

$$
\begin{aligned}
F_{2}(s) & =C(s)\left(e^{-\tau s} X_{1}(s)-X_{2}(s)\right) \\
& =\frac{\left(C(s) e^{-\tau s}+\left(1+P(s) C(s)-P(s) C(s) e^{-2 \tau s}\right)\right) P(s)\left(F_{h}(s)+F_{e}(s)\right)}{\left(1+P(s) C(s)+P(s) C(s) e^{-\tau s}\right)\left(1+P(s) C(s)-P(s) C(s) e^{-\tau s}\right)} .
\end{aligned}
$$

In particular, when $F_{e}=0$ 
$\left[\begin{array}{c}E(s) \\ F_{2}(s)\end{array}\right]=\left(\frac{T(s)}{1+T(s) e^{-\tau s}}\right)\left[\begin{array}{c}1 / C(s) \\ \frac{e^{-\tau s}}{1+P(s) C(s)\left(1-e^{-\tau s}\right)}\end{array}\right] F_{h}(s)$,

where $T(s)=P(s) C(s)(1+P(s) C(s))^{-1}$. Therefore, optimal gains from the $H_{\infty}$ control point of view are the ones which solve the problem

$\min _{K_{p}, K_{d}}\left\|\frac{P(s)}{1+P(s) C(s)\left(1+e^{-\tau s}\right)}\left[\begin{array}{c}\rho \\ \frac{C(s)}{\left(1+P(s) C(s)\left(1-e^{-\tau s}\right)\right)}\end{array}\right]\right\|_{\infty}$,

where $\rho$ is a design parameter which represents the "trade-off" between small tracking error $e$ and small control action $F_{2}$. Depending on the values of $\rho$, the optimal $K_{p}$ and $K_{d}$ are obtained, for each fixed $m=1, b=0.1$ and $\tau=0.05$, as shown in Table 1 .

It is easy to see that for large values of $\rho$ (emphasizing tracking performance, i.e., trying to make $\|e\|_{2}$ small compared to $\left\|F_{2}\right\|_{2}$ ) $H_{\infty}$ optimal gains are in the order of $K_{p} \in[240,310]$ and $K_{d} \in[40,55]$. The next subsection includes a comparison between this set of values and another set of gains obtained from a different optimality criterion.

\subsection{Stability margin optimization}

Introduce now: $\alpha:=\left(b K_{d}\right) /\left(m K_{p}\right)$ and assume that $\alpha<1$. Let $\omega_{p}$ be the smallest $\omega>0$ satisfying

$\tan ^{-1}(\alpha \omega)=\tan ^{-1}(\omega)-\frac{h \omega}{2}=-\pi$,

where $h=\left(\left(\tau_{1}+\tau_{2}\right) b\right) /(2 m)$.

As mentioned in the proof of Theorem 1 (see Appendix A), one of the stability conditions is

$\left(\frac{b^{2}}{m K_{p}}\right)\left(\frac{1+\omega_{p}^{2}}{2(1-\alpha)}\right)>1$.

Table 1

$H_{\infty}$ optimal gains for different $\rho$.

\begin{tabular}{rrrrrrr}
\hline$b^{2} \rho$ & 0.01 & 0.1 & 1 & 10 & 50 & 100 \\
\hline$K_{p}$ & 0.8 & 17.1 & 85.0 & 246 & 305 & 310 \\
$K_{d}$ & 8.8 & 10.2 & 15.2 & 43 & 55 & 51 \\
\hline
\end{tabular}

Table 2

Optimal gains and $G M_{1}$ for different $\rho_{2}$, when $\tau=0.05, m=1$, and $\rho_{1}=b^{2}=0.01$.

\begin{tabular}{|c|c|c|c|c|c|c|c|c|}
\hline$\rho_{2}$ & 10 & 20 & 30 & 40 & 50 & 60 & 80 & 100 \\
\hline$K_{p}$ & 94 & 207 & 301 & 389 & 425 & 436 & 446 & 453 \\
\hline$K_{d}$ & 2.4 & 6.3 & 12.7 & 34.3 & 82 & 127 & 207 & 291 \\
\hline$G M_{1}$ & 1.33 & 2.9 & 4.2 & 5.5 & 6.0 & 6.1 & 6.16 & 6.2 \\
\hline
\end{tabular}

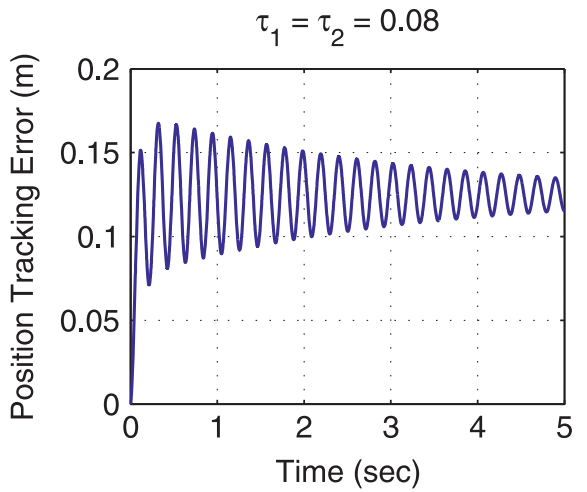

Note that $\omega_{o}<\omega_{p}$ so, defining

$G M_{1}:=\left(\frac{b^{2}}{m K_{p}}\right)\left(\frac{1+\omega_{o}^{2}}{2(1-\alpha)}\right)$,

then $G M_{1}>1$ implies (28). So, one will try to make $G M_{1}$ as large as possible. On the other hand, for large bandwidth in the system (fast response) it is required that $\omega_{c}$ is as large as possible, i.e.

$\omega_{c}^{2}+1=\frac{m K_{p}}{b^{2}} 2(1-\alpha)$

should be as large as possible. But this conflicts with $G M_{1}$ should be large condition. So, blending these two conflicting objectives and trying to

maximize $\min \left\{\rho_{1}\left(\omega_{c}^{2}+1\right), \frac{1}{\rho_{1}} G M_{1}\right\}$,

where $\rho_{1}$ assigns a relative weight for each component of the problem. The solution of this problem gives

$\frac{m K_{p}}{b^{2}}=\frac{1}{\rho_{1}} \frac{\sqrt{1+\omega_{o}^{2}}}{2(1-\alpha)}$

Under this choice, it follows:

$G M_{1}=\rho_{1} \sqrt{1+\omega_{o}^{2}}$.

Note that the right hand sides of (32) and (33) are functions of $\alpha$ once $\rho_{1}$ and $h=\tau b / m$ are fixed.

Now, $\left(m K_{p} / b^{2}\right)$ is the controller gain, and to avoid actuator saturations, this gain should not be too high. So, one can define a new cost function which tries to make $G M_{1}$ large and $K_{p}$ small, the objective here is to minimize the following cost function by appropriately chosen $K_{p}$ :

Cost : $\quad\left(\frac{\rho_{2}}{\rho_{1} \sqrt{1+\omega_{0}^{2}}}+\frac{b^{2}}{m \rho_{2}} \frac{1}{\rho_{1}} \frac{\sqrt{1+\omega_{o}^{2}}}{2(1-\alpha)}\right)$,

where $\rho_{2}$ assigns relative weights for $G M_{1}$ and $K_{p}$. Note that $\rho_{1}$ does not play a role in the solution of (34). Once $\rho_{2}$ and $h=\tau b / m$ are fixed, the cost function defined in (34) depends on $\alpha$ only. Minimizing the cost function gives optimal $\alpha$, then this gives $\omega_{0}$ and $K_{p}$ via (32); and once $K_{p}$ is known, $K_{d}=\alpha m K_{p} / b$ can be found.

Table 3

Allowable perturbations of delay for $H_{\infty}$ optimal gain parameters when $m=1$ and $b=0.1$.

\begin{tabular}{lllcccc}
\hline$K_{p}$ & 17.1 & 85.0 & 246 & 305 & 310 & 400 \\
$K_{d}$ & 10.2 & 15.2 & 43 & 55 & 51 & 40 \\
$\tau_{\max }$ & 0.458 & 0.181 & 0.120 & 0.110 & 0.108 & 0.087 \\
\hline
\end{tabular}

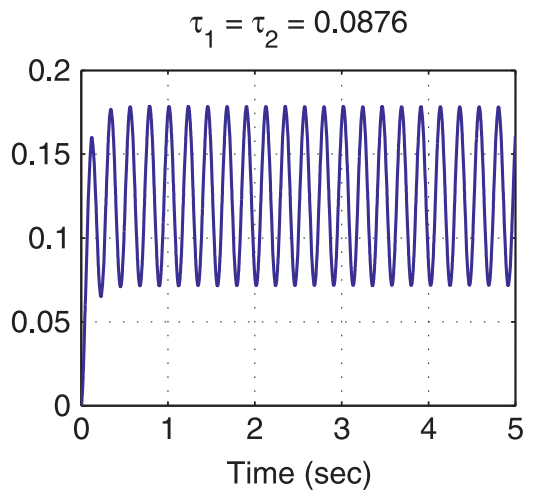

Fig. 8. System is stable for $\tau<0.0876$, marginally stable for $\tau=0.0876$ and unstable for $\tau>0.0876$ when $K_{p}=400$ and $K_{d}=40$. 
Table 2 shows the optimal gains for varying $\rho_{2}$ when $\rho_{1}=b^{2}=$ $0.01, m=1$ and $h=\tau b / m=0.005$ are fixed.

Table 2 shows that $G M_{1}$ increases with increasing $\rho_{2}$, but for $\rho_{2} \geq 50$ additional gain in $G M_{1}$ is very small. Therefore, a meaningful choice would be $K_{p} \in[390,410]$ and $K_{d} \in[35,45]$. Compared to the $H_{\infty}$ optimal gains corresponding to relatively large $\rho$ values, the above $K_{p}$ values are about $1.3-1.5$ times higher, whereas $K_{d}$ values are 1.14-1.25 times lower. For the experimental tests, the values $K_{p}=400$ and $K_{d}=40$ are used and results are reported in the next section. These correspond to $\rho_{2} \approx 42$ in the above table. For the $H_{\infty}$ optimal gains one may select $K_{p}=275$ and $K_{d}=45$; the stability margins are expected to be larger in this case, but the response will be slower. For relatively small $\rho$ values in the $H_{\infty}$ optimal design, i.e. $K_{p}=85$ and $K_{d}=15$ (e.g. for $b^{2} \rho=1$ ) in which case the emphasis on tracking performance is diminished compared to larger $\rho$ values. In the next section, experimental results for the above mentioned parameters are illustrated.

\section{Robustness analysis}

\subsection{Delay perturbations}

Smallest time delay which destabilizes the feedback system for a given set of controller and plant parameters can be calculated using Theorem 1 . This can be seen as the largest tolerable delay. Time-domain simulation in Fig. 8 illustrates the results found in Table 3 (Fig. 9).

\subsection{Parametric plant perturbations}

\section{Introducing}

$C(s):=C_{1}(s)=C_{2}(s), \quad L_{1}(s):=P_{1}(s) C(s), \quad L_{2}(s):=P_{2}(s) C(s)$,

leads to the characteristic equation of the form

$1+L_{1}(s)+L_{2}(s)+L_{1}(s) L_{2}(s)-L_{1}(s) L_{2}(s) e^{-2 \tau s}=0$.
After some algebraic manipulations, the characteristic equation can be written as

$\frac{1}{P_{1}(s) s}=m_{1} s+b_{1}=\frac{\left(1+L_{2}(s)-L_{2}(s) e^{-2 \tau s}\right) C}{-\left(1+L_{2}(s)\right)}=: H(s)$.

Parameters pairs $m_{1}^{*}$ and $b_{1}^{*}$ may be found for marginally stable characteristic equation (37) as in Morarescu, Niculescu, and Gu (2010).

$m_{1}^{*}=\frac{\operatorname{Im}(H(j \omega))}{\omega}, \quad b_{1}^{*}=\operatorname{Re}(H(j \omega)) \forall \omega$.

Fig. 9 shows the allowable parameter region determined from (38), as well as time domain responses for two different choices of the parameters.

\subsection{Robustness against unmodeled dynamics}

The plant model used can be slightly different than the real model due to uncertainties such as unmodeled dynamics and approximation of the parameters. To avoid undesirable effects of these uncertainties, the controller gains used should stabilize all possible plants. Defining one of the plants as

$P_{1}(s)=P(s)+\Delta(s)$,

the robust stability test may be applied. Characteristic equation of the perturbed system is

$(1+P(s) C(s))(1+(P(s)+\Delta) C(s))-(P(s)+\Delta) P(s) C(s)^{2} e^{-2 \tau s}=0$.

After some algebraic manipulations, characteristic equation becomes the characteristic equation of nominal plant multiplied by a function with perturbed terms.

$$
\begin{aligned}
(1+ & P(s) C(s))\left(1+T(s) e^{-\tau s}\right)\left(1+G(s) f_{\tau}(s)\right)[1+ \\
& \left.+\Delta_{m}\left(\frac{T(s)}{1+T(s) e^{-\tau s}}\right)\left(\frac{1+G(s) f_{2 \tau}(s)}{1+G(s) f_{\tau}(s)}\right)\right],
\end{aligned}
$$

where

$\Delta_{m}(s):=\frac{P_{1}(s)-P(s)}{P(s)} f_{\tau}(s)=\frac{1-e^{-\tau s}}{s}$.

Allowable $m_{1}$ and $b_{1}$ parameters
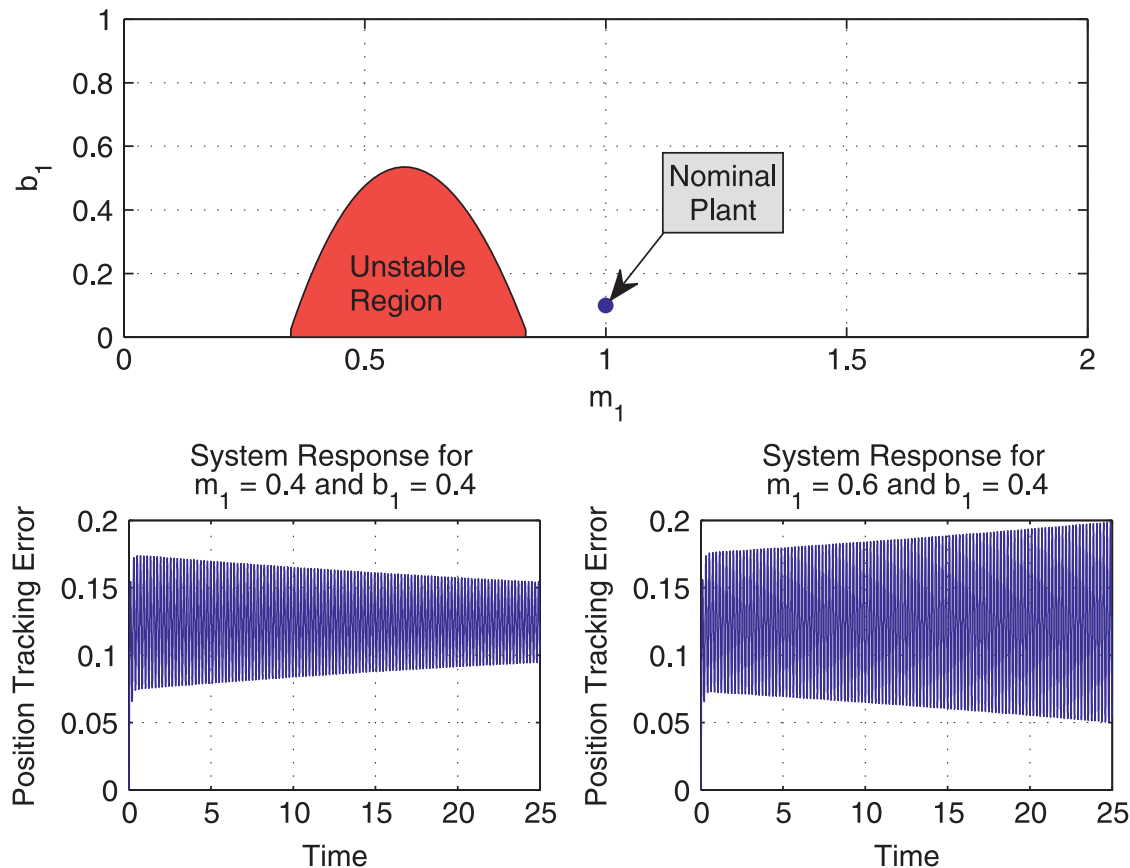

Fig. 9. Allowable plant parameters for $m_{2}=1, b_{2}=0.1, K_{p}=400, K_{d}=40, \tau=0.085$. 
In (41), the transfer functions $T(s)$ and $G(s)$ are as (see also Appendix A)

$G(s)=\frac{K_{p}+K_{d} s}{m s+b}, \quad T(s)=\frac{K_{p}+K_{d} s}{s(m s+b)+K_{p}+K_{d} s}$,

and $\Delta_{m}$ is called multiplicative perturbation. In "Optimal Gains" section, controller parameters are provided for which the nominal feedback system is stable and performance criteria is satisfied. For robust stability, these parameters should also satisfy following inequality:

$$
\begin{gathered}
\left\|\Delta_{m}(s)\left(\frac{T(s)}{1+T(s) e^{-\tau s}}\right)\left(\frac{1+G(s) f_{2 \tau}(s)}{1+G(s) f_{\tau}(s)}\right)\right\|_{\infty} \\
:=\left\|\Delta_{m}(s) R(s)\right\|_{\infty}<1 .
\end{gathered}
$$

By using Eq. (44), the allowable magnitude of perturbation can be derived

$$
\left|\Delta_{m}(j \omega)\right|<\frac{1}{|R(j \omega)|} .
$$

Fig. 10 shows that the only frequency range where tolerable uncertainty bound is less than $100 \%$ is between $20 \mathrm{rad} / \mathrm{s}$ and $50 \mathrm{rad} / \mathrm{s}$ (where tolerable uncertainty bound is between $50 \%$ and 100\%); any unmodeled lightly damped flexible modes in this

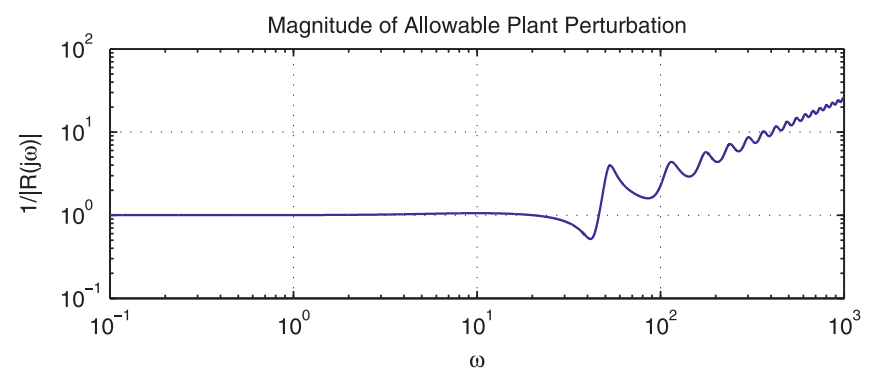

Fig. 10. $m=1, b=0.1, \tau=0.05, K_{p}=400, K_{d}=40$.
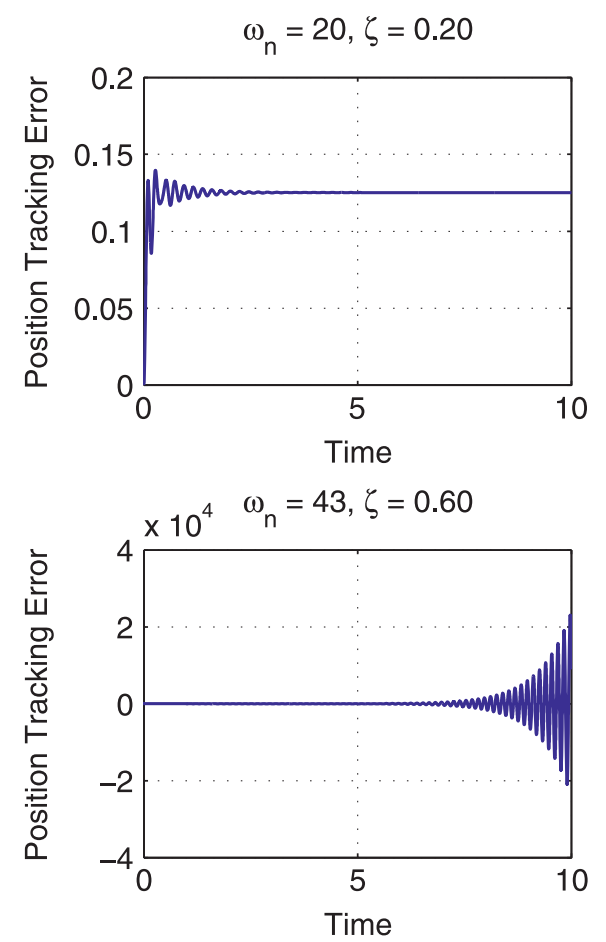
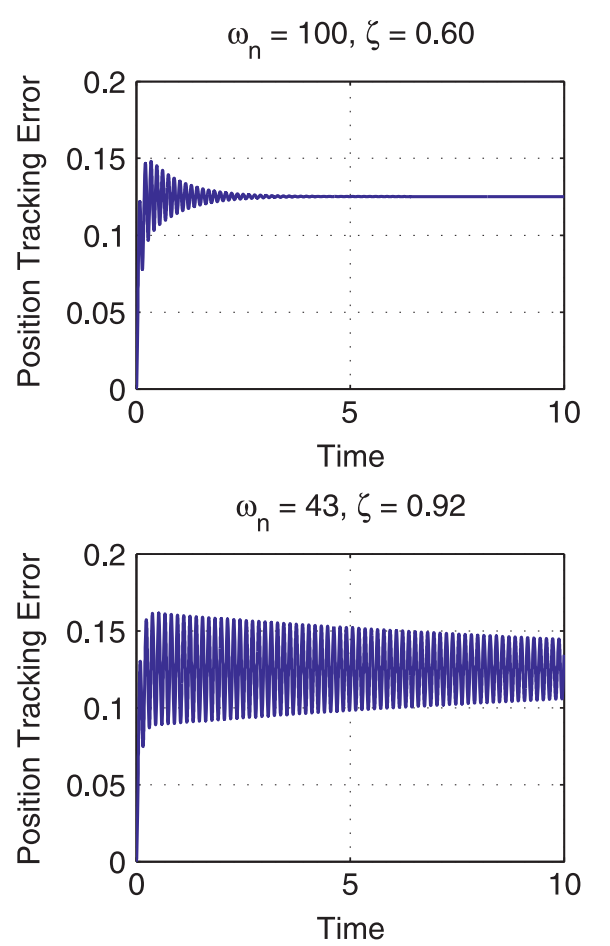

frequency range may destabilize the feedback system, otherwise the system is quite robust to unmodeled dynamics.

To illustrate this result, the system is perturbed with

$W(s)=\frac{\omega_{n}^{2}}{s^{2}+2 \zeta \omega_{n} s+\omega_{n}^{2}}$,

which represents an unmodeled flexible mode of the system. The perturbed plant is defined as follows:

$P_{1}(s)=P(s)+P(s) W(s)$.

Corresponding simulation results with different $\zeta$ and $\omega_{n}$ are shown in Fig. 11.

\section{Experimental results}

\subsection{Experimental setup}

In order to guarantee a full control of the communication time-delays and processing time, all the control algorithms (for haptic interface/virtual object) and virtual environment simulations will be run on the same computer.

The haptic interface, Fig. 12a and b, consists of three direct-drive motor and three optical quadrature encoder with $1000 \mathrm{pts} / \mathrm{rev}$ (with a gear ratio of $1 / 10$ ). The controllers and the virtual simulation are running in real time mode (on RTAI Linux) with a sampling time of $1 \mathrm{~ms}$. Fig. 12c illustrates the virtual scene and the virtual object.

The virtual object is modeled to be some spherical mass (equal to the haptic interface mass) $\left(M_{h}=M_{v}\right)$. The environmental force $\left(F_{e_{x}}, F_{e_{y}}, F_{e_{z}}\right)$ resulting in case of an impact with the virtual environment is defined by the following equation:

$F_{e}=K_{\text {wall }}\left(P_{v}-P_{\text {wall }}\right)+B_{\text {wall }} \dot{P}_{v}$,

with

$F_{e}=\left(\begin{array}{c}F_{e_{x}} \\ F_{e_{y}} \\ F_{e_{z}}\end{array}\right), \quad P_{v}=\left(\begin{array}{c}x_{v} \\ y_{v} \\ z_{v}\end{array}\right), \quad P_{\text {wall }}=\left(\begin{array}{c}x_{\text {wall }} \\ y_{\text {wall }} \\ z_{\text {wall }}\end{array}\right)$,

Fig. 11. $m=1, b=0.1, \tau=0.05, K_{p}=400, K_{d}=40$. 


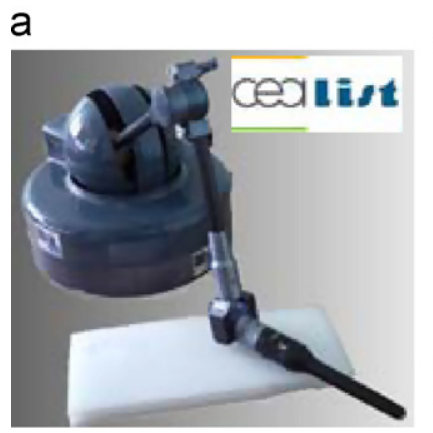

b

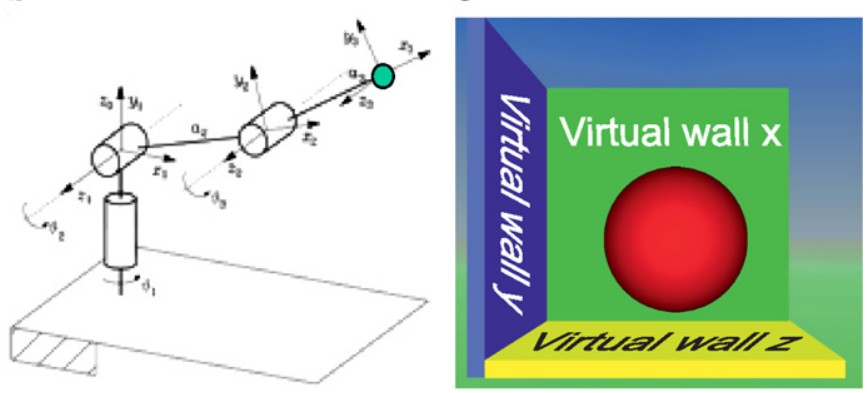

Fig. 12. Haptic system. (a) Haptic interface. (b.) Robot geometry. (c) Virtual scene.
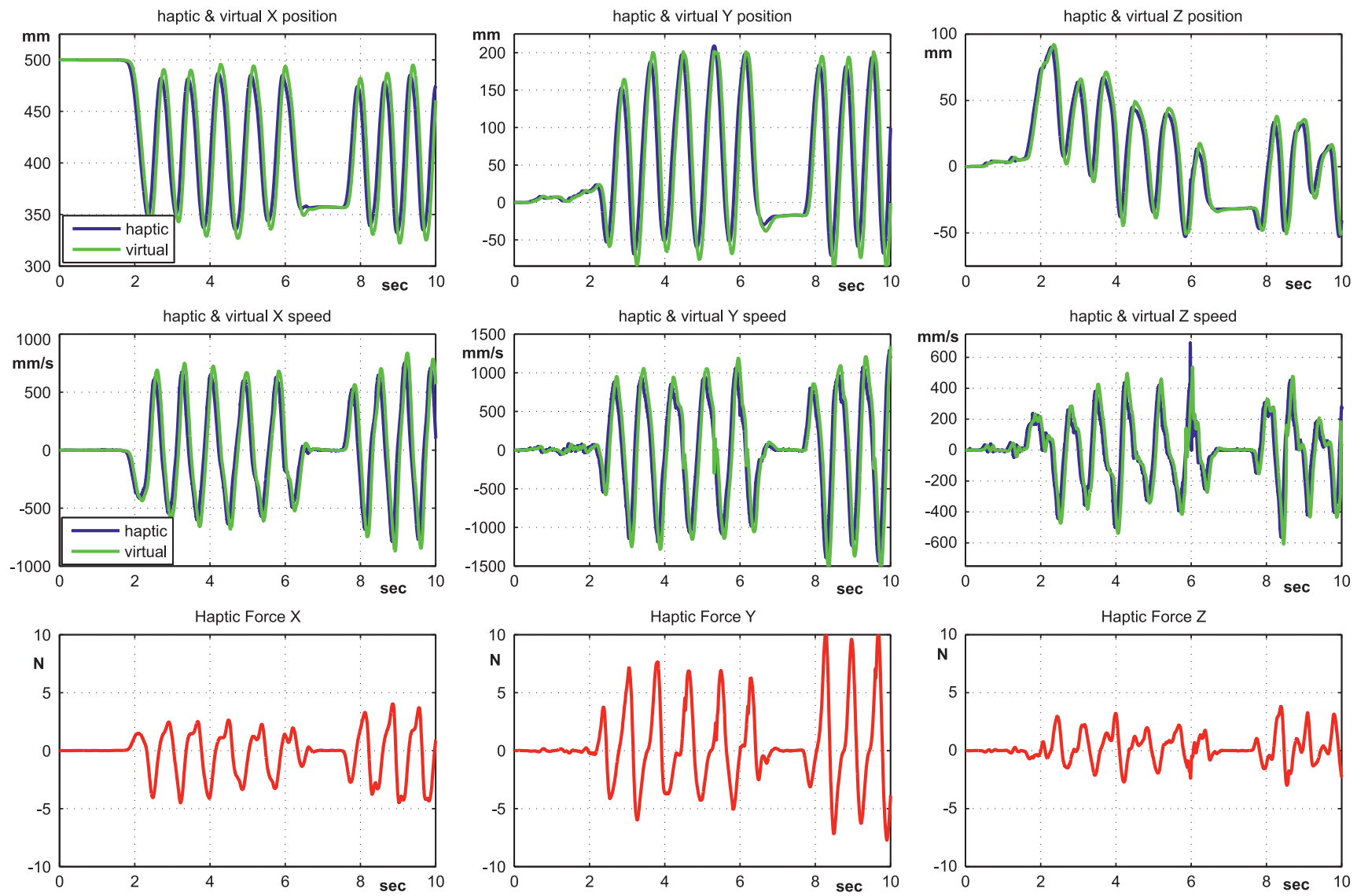

Fig. 13. Free motion for $K_{p}=85$ and $K_{d}=15$.

where $K_{\text {wall }}=20000$ and $B_{\text {wall }}=10$ represent the stiffness and the damping used to compute the virtual force environment, $P_{\text {wall }}$ is the virtual wall position $(x, y, z)$ and $P_{v}, \dot{P}_{v}$ are the virtual object position and velocity, respectively.

The testing scenarios are the same for each experimental category:

- free motion (random motions on each axis)

- restricted motion (wall contact on each axis).

\subsection{Results}

The haptic systems must be analyzed in two distinct situations: free and restricted motion, respectively. A constant timedelay $\tau_{1}=\tau_{2}=50 \mathrm{~ms}$ will be considered for all the experiments.
In Fig. 13, it is presented the free motion case for $K_{p}=85, \quad K_{d}=15$,

as discussed in Table 1.

The obtained results are "good", in the sense that the curves corresponding to the haptic interface and virtual object are almost identical, which shows a low tracking error. The system appears to be stable in closed-loop and robust to perturbations and the force feedback is small, i.e. the viscosity effect is low.

Next, in Fig. 14, using the same gains, the results for the restricted motion case are presented.

As expected, the tracking error is important and the contact effect felt by the end user is low, because the tuning strategy is contradictory. More precisely, for "good" results in free motion, small gains are desired (exactly what was obtained), but in restricted motion, in order to have a small tracking error and a stiff response, high gains are explicitly needed. 

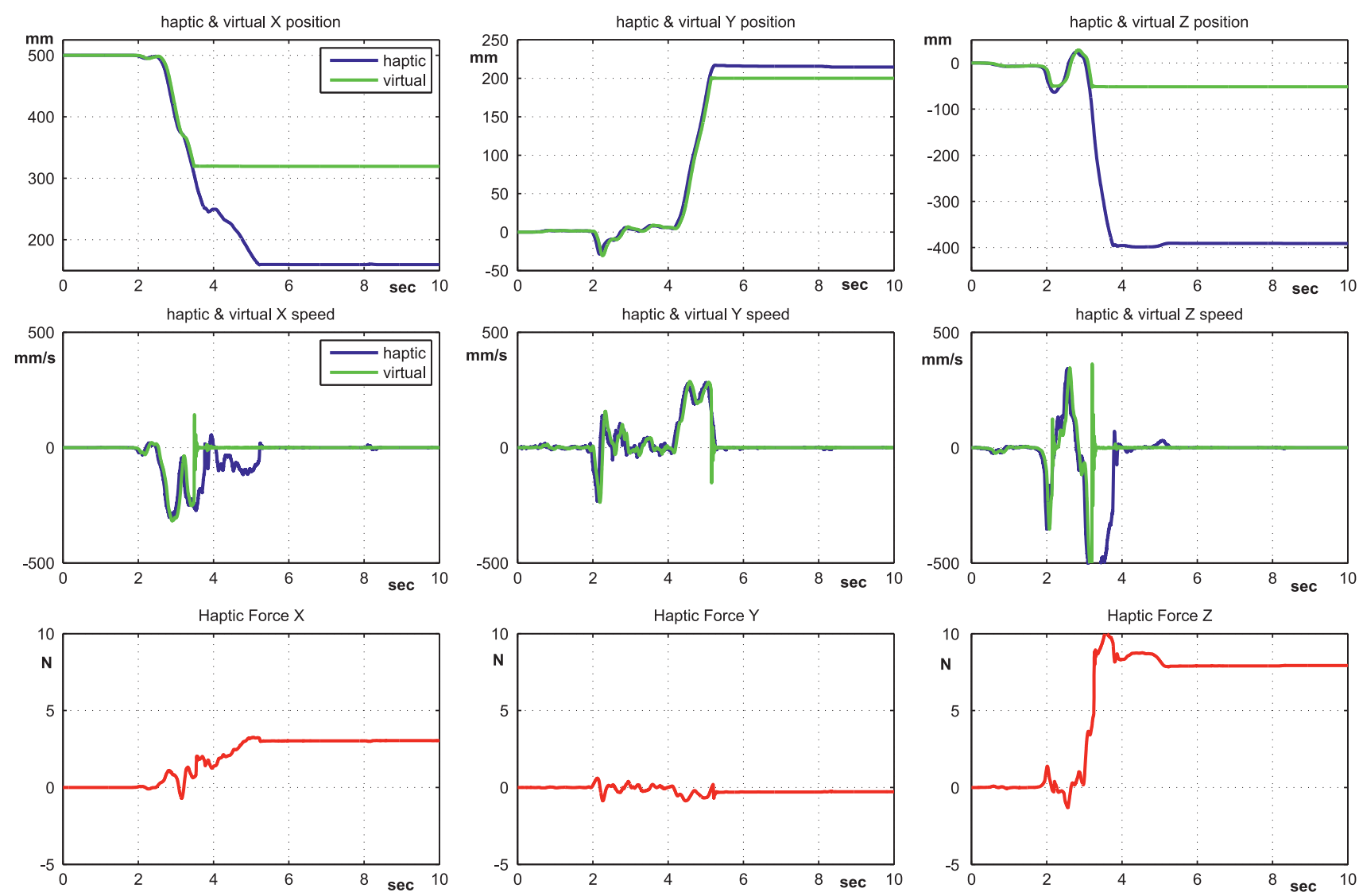

Fig. 14. Restricted motion for $K_{p}=85$ and $K_{d}=15$.

In order to decrease the tracking error and to provide a more accurate contact feeling in restricted motion case, the following values will be used (as presented in Table 1):

$K_{p}=250, \quad K_{d}=45$.

Figs. 15 and 16 presents the results in free and restricted motion for the new values of the PD gains.

In free motion, it can be observed that there is a slightly degradation with respect to the previous example in terms of force feedback, i.e. the force is more important. The viscosity effect is still low, the manipulation can be made in a pleasant way without feeling a disturbing force. From the perspective of tracking error, the performances are good, as the curves demonstrate, similar to the previous case. In restricted motion, there is an important amelioration compared to the previous case, but still the performances are not the desired ones. More precisely, the impact moment is not sufficiently stiff in order to provide to the end user an accurate contact feeling. The overall performances of this example are better than the previous one.

Further on, Figs. 17 and 18 present the results in free and restricted motion for

$K_{p}=400, K_{d}=40$,

as proposed in Section 4.2 .

In free motion, the viscosity effect is more important and it appears to be less pleasant to manipulate than the previous case, but in restricted motion the tracking error is considerably lower and the response is stiffer. As the curves illustrate, in free motion the tracking error is low, i.e. the performances are good as in the previous cases.

As expected, for good results in free motion, small gains are required and for restricted motion, high gains are desired. Any "trade-off" made in one sense or another will result in some overall performance degradation.

In order to validate the theoretical result obtained here on stability, the gains were pushed over the limit of stability and in Fig. 19 is presented an unstable behavior of the system.

More precisely, for $K_{d}=40$, the maximum allowable $K_{p}$ is about 1000. Considering the model uncertainties, the system's frictions and the operator's hand the system is still stable at this value. Another reason is that it is difficult to obtain high frequencies and the haptic interface input. Starting from $K_{p}=1100$ the system becomes unstable.

\section{Conclusions}

In this paper, a complete stability analysis for a bilateral haptic system coupled to a virtual environment and affected by timedelays is presented.

First, appropriate necessary and sufficient condition have been derived to guarantee the closed-loop stability. Such conditions are analytical and allow an easy characterization of the stability regions in the controller parameter-space. Next, using optimization techniques and based on the stability limits, optimal controllers from the tracking error point of view are proposed. More precisely, the PD gains are tuned according to a maximum allowed tracking error. Furthermore, a robustness analysis is performed in order to highlight the limitations in terms of maximum time-delay, parametric plant perturbations and unmodeled dynamics.

To obtain good performance from the transparency point of view in free and restricted motion, using the same PD gains, a compromise must be made in order to guarantee minimal 

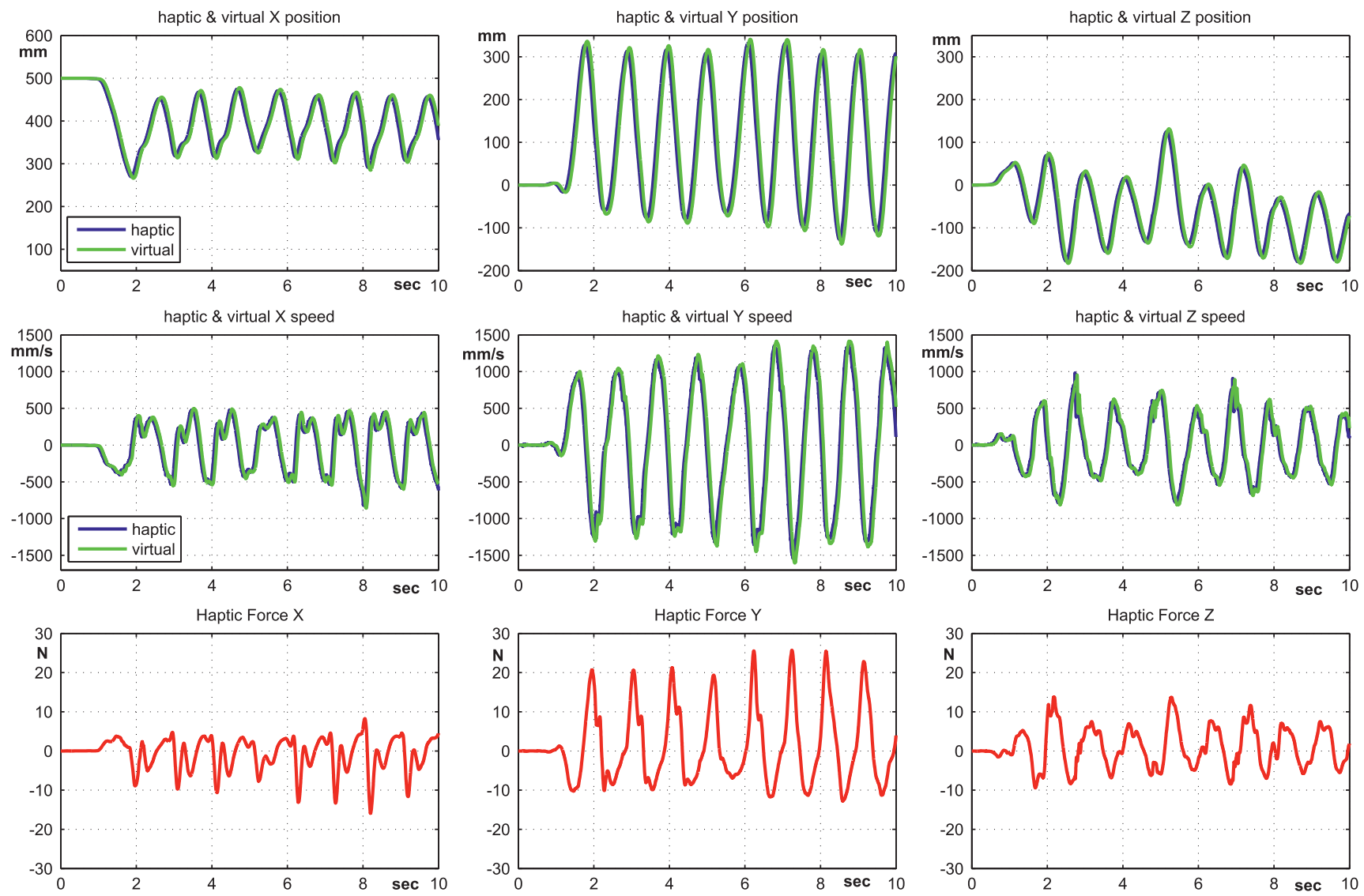

Fig. 15. Free motion for $K_{p}=250$ and $K_{d}=45$.
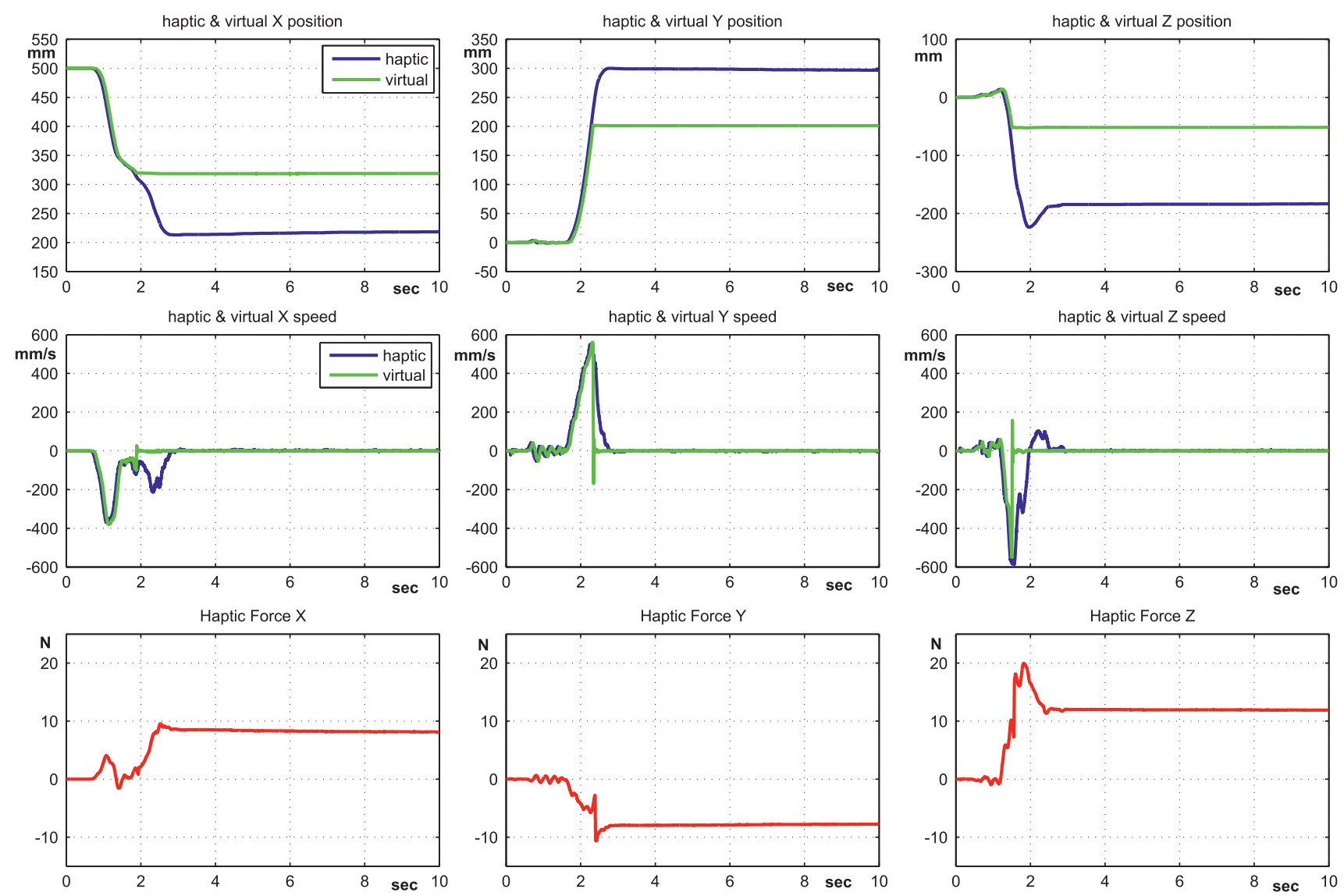

Fig. 16. Restricted motion for $K_{p}=250$ and $K_{d}=45$. 

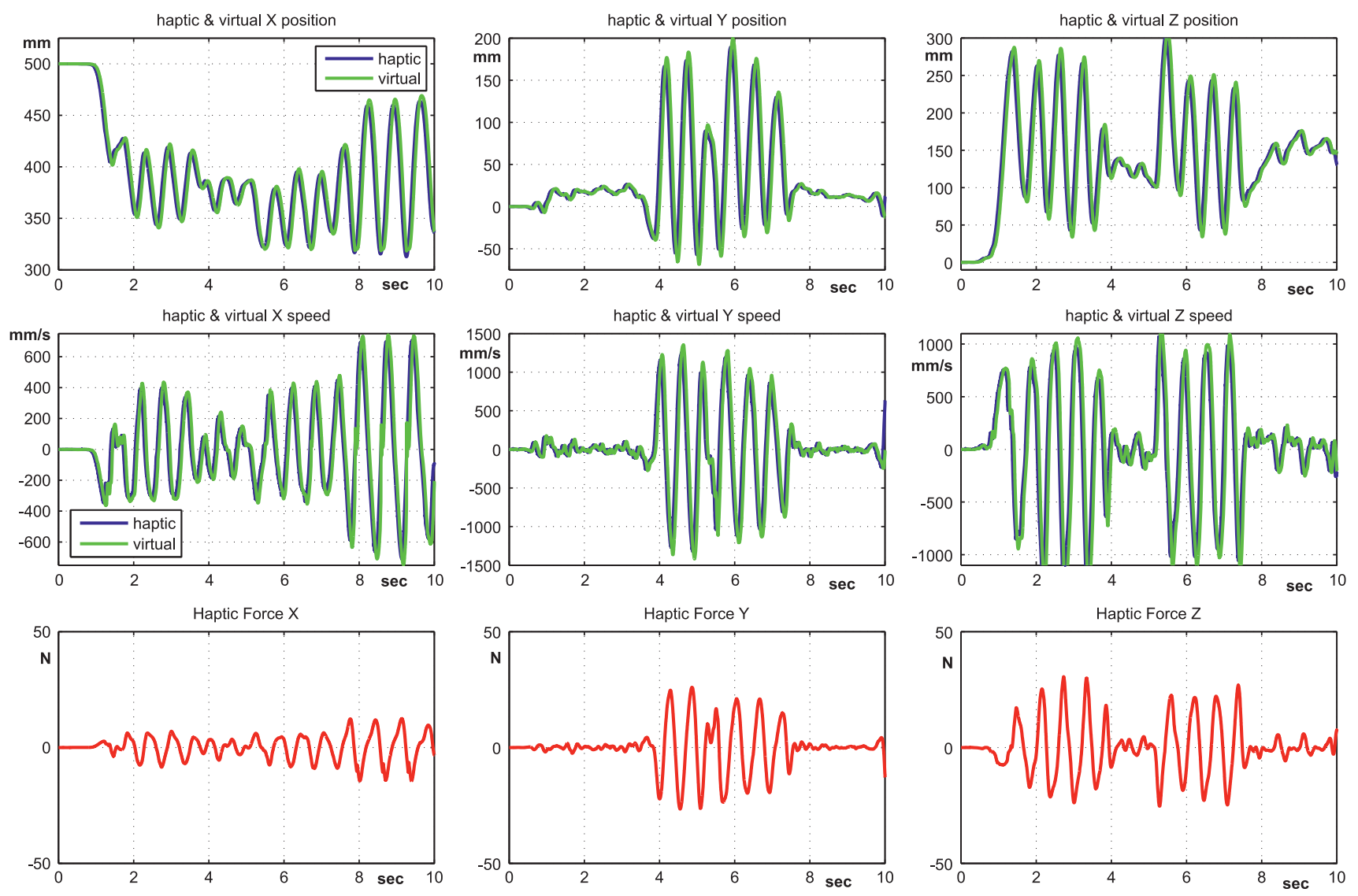

Fig. 17. Free motion for $K_{p}=400$ and $K_{d}=40$.
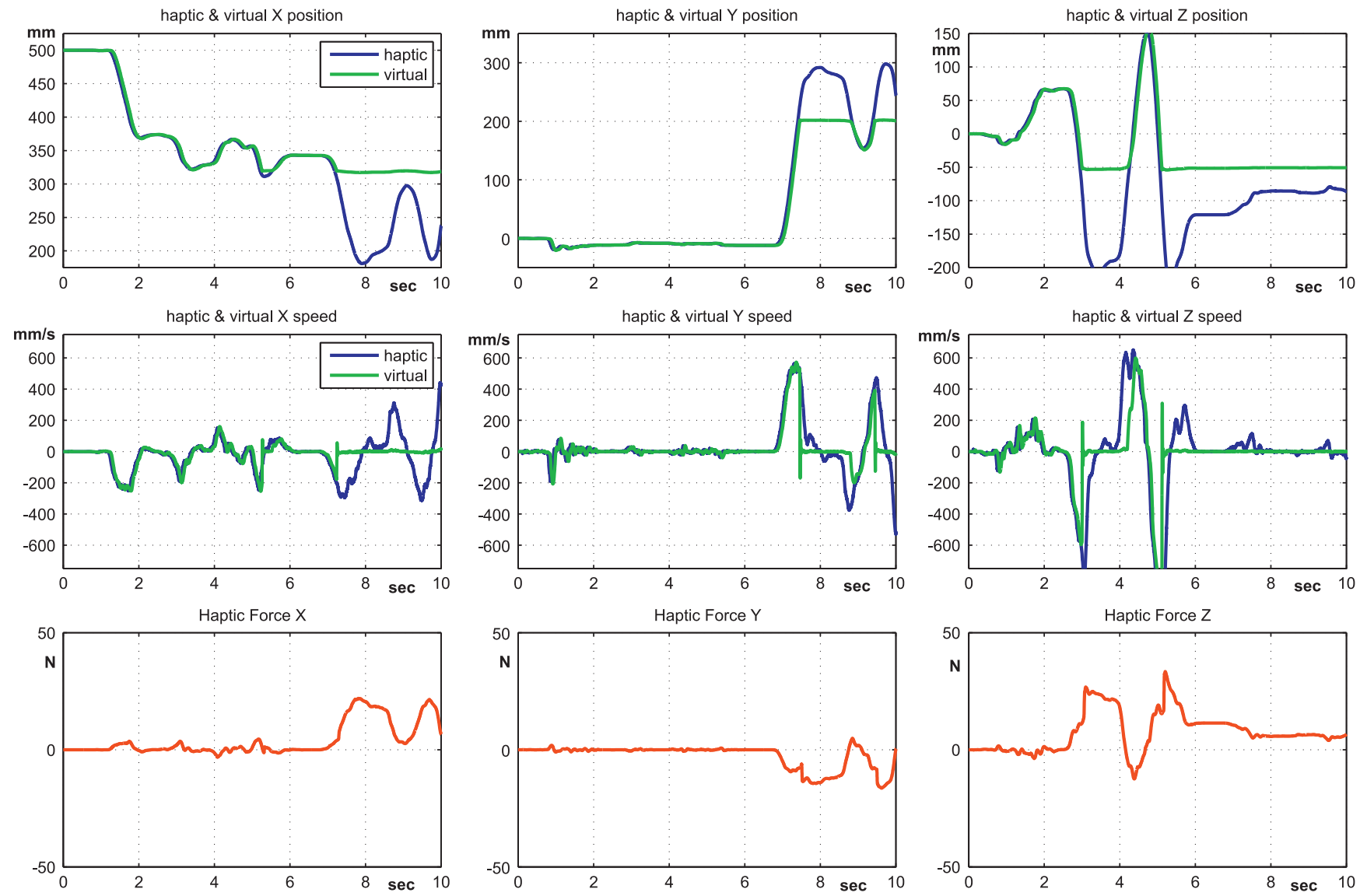

Fig. 18. Restricted motion for $K_{p}=400$ and $K_{d}=40$. 

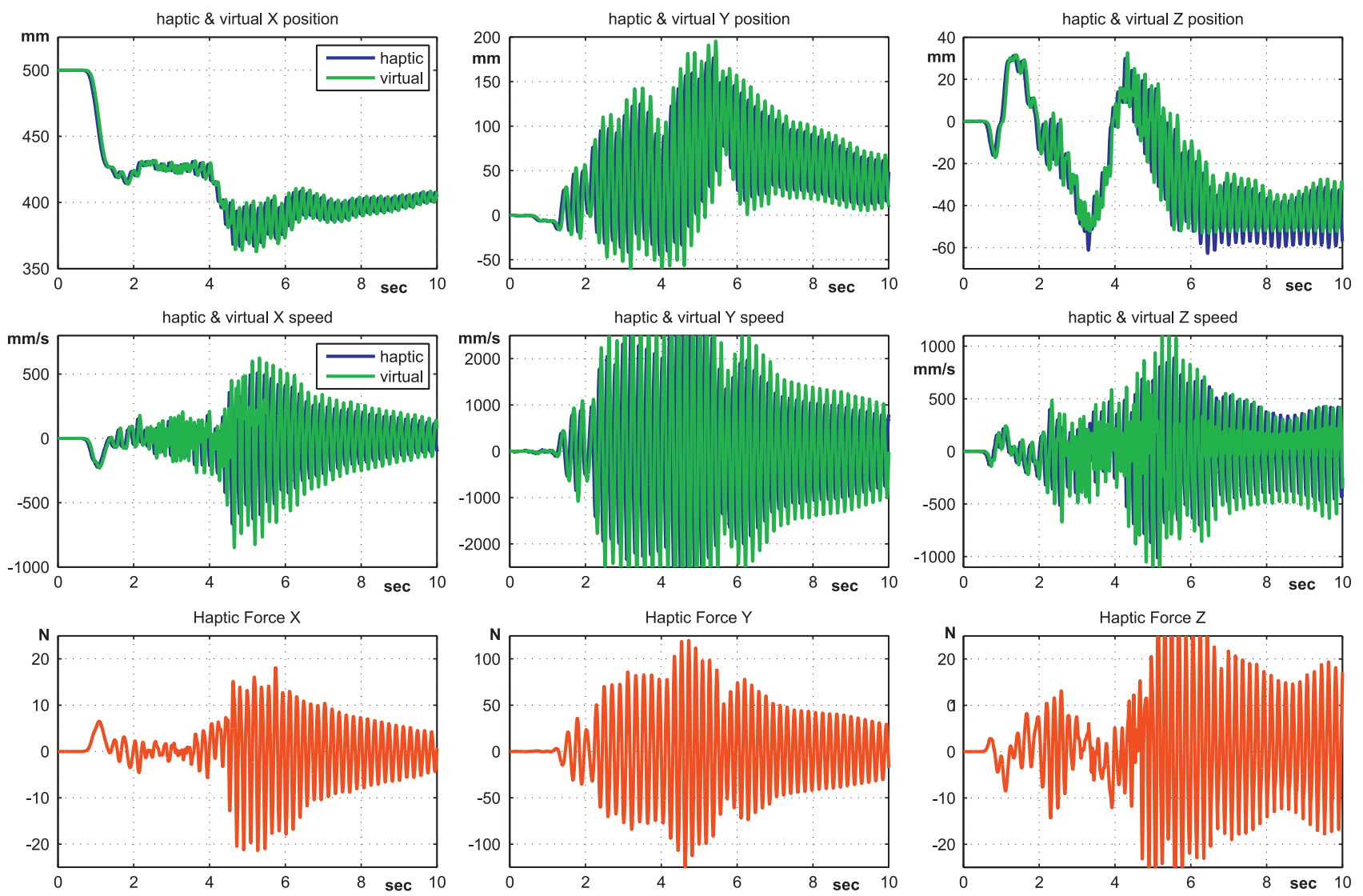

Fig. 19. Unstable behavior for $K_{p}=1100$ and $K_{d}=40$.

performance in both cases. The proposed controllers have been tested and validated on a 3 degree-of-freedom haptic system in free and restricted motions. Another solution is to use a gain scheduling approach in order to switch from small to high gains depending on the case. A special attention is needed for this approach since both controllers must be updated, and since the system is affected by time-delays, there is a "critical" moment when the gains will be different at each side, moment that can induce unwanted effects and behaviors. The stability analysis in this case would fall into the framework of switched time-delays systems and stability can be guaranteed for a sufficiently large dwell time, see for example Çalışkan, Özbay, and Niculescu (2011), Yan and Özbay (2008), Yan, Özbay, and Șansal (2011) and their references.

\section{Acknowledgments}

The authors wish to thank associate editor and anonymous reviewers for their useful comments and remarks that helped us to improve the overall contribution of the paper.

This work is supported in part by the French-Turkish PIA Bosphorus (TUBITAK Grant no. 109E127 and EGIDE Project no. 22974WJ). The work of Bogdan Liacu was financially supported by CEA LIST, Interactive Robotics Laboratory BP 6, 18 route du Panorama, F-92265 Fontenay-aux-Roses, France. Hitay Özbay and Ahmet Taha Koru acknowledge partial financial support by DPTHAMIT project.

\section{Appendix A. Reduction of the stability conditions}

Since $(1+P C)^{-1}$ is a stable transfer function, from (12) it is worth mentioning that the feedback system is stable if and only if the following two equations do not have zeros in $\mathbb{C}_{+}=$ $\{s: \operatorname{Re}(s) \geq 0\}$ :

$1+G(s)\left(\frac{1-e^{-\tau s}}{s}\right)=0, \quad$ where $G(s)=\frac{K_{p}+K_{d} s}{m s+b}$,

$1+T(s) e^{-\tau s}=0, \quad$ where $T(s)=\frac{K_{p}+K_{d} s}{s(m s+b)+K_{p}+K_{d} s}$.

Now define

$K:=\frac{K_{p}}{b}, \quad \tau_{c}:=\frac{K_{d}}{K_{p}}, \quad \tau_{p}:=\frac{m}{b}$,

then $G(s)$ and $T(s)$ can be re-written as

$G(s)=K \frac{1+\tau_{c} s}{1+\tau_{p} s}$

$T(s)=\frac{K\left(1+\tau_{c} s\right)}{\tau_{p} s^{2}+\left(1+\tau_{c} K\right) s+K}$.

Further, a frequency normalization can be made

$\widehat{s}=\tau_{p} s$,

and introduce new definitions

$L:=\frac{1}{K \tau_{p}}=\frac{b^{2}}{m K_{p}}, \quad \alpha:=\frac{\tau_{c}}{\tau_{p}}=\frac{b K_{d}}{m K_{p}}, \quad h:=\frac{\tau}{\tau_{p}}=\frac{\left(\tau_{1}+\tau_{2}\right) b}{2 m}$,

so that the characteristic Eqs. (A.1) and (A.2) can be re-written as

$1+\frac{1}{L} \frac{(1+\alpha \widehat{S})}{(1+\widehat{S})}\left(\frac{1-e^{-\widehat{h}}}{\widehat{S}}\right)=0$, 


$$
1+\frac{(1+\alpha \widehat{S})}{\left(L \widehat{S}^{2}+(L+\alpha) \widehat{S}+1\right)} e^{-\widehat{h}}=0 .
$$

The next step is to find the controller parameters $L$ and $\alpha$ (which define $K_{p}$ and $K_{d}$ ), as functions of $h$, that place all the roots of (7) and (8) in $\mathbb{C}_{\text {. }}$. In what follows without any lack of generality only the case where $K_{p}$ and $K_{d}$ are positive, i.e., $L>0$ and $\alpha>0$ is considered. It is worth mentioning that, in practice, such a situation occurs most of the cases.

Analysis of stability conditions of transfer functions (A.7) and (A.8) are based on Nyquist Stability Criterion. Let us consider (A.7) first. Since $\left|e^{-j h \omega}\right|=1$ for all $\omega \in \mathbb{R}$, the phase of $\left(1-e^{-j h \omega}\right)$ is between $+\pi / 2$ and $-\pi / 2$ for all $\omega>0$ and

$\lim _{\omega \searrow 0^{+}} \angle\left(1-e^{-j h \omega}\right)=+\frac{\pi}{2}$.

Therefore,

$0 \leq \angle f(j \omega) \leq-\pi, \quad \forall \omega \in \mathbb{R}, \quad$ where $f(\widehat{s}):=\frac{1-e^{-\widehat{h s}}}{\widehat{s}}$.

This means that if $\alpha>1$, the phase of $(1+j \alpha \omega) /(1+j \omega) f(j \omega)$ is always strictly greater than $(-\pi)$ for all $\omega \geq 0$. Thus, all the roots of (A.7) are in $\mathbb{C}$. when $\alpha>1$, independent of $L$ and $h$. Furthermore, when $\alpha=1$ Eq. (A.7) reduces to

$1+\frac{1}{L} f(S)=0$.

Note that whenever $\angle f(j \omega)=-\pi,|f(j \omega)|=0$ holds. This fact, together with (A.10), implies that when $\alpha=1$ all the roots of (A.7) are in $\mathbb{C}_{-}$, independent of $L$ and $h$. In conclusion, the analysis of (A.7) becomes interesting only if $\alpha<1$. In this case, all the roots of (A.7) are in $\mathbb{C}_{\text {. }}$ if and only if the following condition is met:

$L>\left|\frac{1+j \alpha \omega_{p}}{1+j \omega_{p}}\right|\left|f\left(j \omega_{p}\right)\right|$,

where $\omega_{p}$ is the smallest $\omega>0$ satisfying

$\tan ^{-1}(\alpha \omega)-\tan ^{-1}(\omega)-\frac{h \omega}{2}=-\pi$.

The condition (A.12) gives an allowable region in the $(\alpha, L)$ plane for all the roots of (A.7) to be in $\mathbb{C}_{\text {. }}$ when $\alpha<1$. Since,

$f(j \omega)=\frac{1-e^{-j \omega h}}{j \omega}=\frac{\sin (\omega h)}{\omega}-j \frac{(1-\cos (\omega h))}{\omega}$

the following identity used in (A.13) can be derived as follows:

$\angle f(j \omega)=\tan ^{-1}\left(\frac{\cos (\omega h)-1}{\sin (\omega h)}\right)=-\frac{h \omega}{2} \quad \forall \omega \in\left[0, \frac{2 \pi}{h}\right]$,

by using half-angle formulas, followed by simplification using the trigonometric identity $\cos ^{2}(\omega h / 2)=1-\sin ^{2}(\omega h / 2)$.

Eq. (A.13) can be re-arranged for $\alpha<1$ as

$\pi-\left(\tan ^{-1}\left(\omega_{p}\right)-\tan ^{-1}\left(\alpha \omega_{p}\right)\right)=\frac{h \omega_{p}}{2}$.

It is a simple exercise to show that

$\left|f\left(j \omega_{p}\right)\right|=\frac{\sin \left(h \omega_{p} / 2\right)}{\omega_{p} / 2}=\frac{2(1-\alpha)}{\sqrt{(1-\alpha)^{2} \omega_{p}^{2}+\left(1+\alpha \omega_{p}^{2}\right)^{2}}}$.

Using this identity, after algebraic manipulations and for $\alpha<1$, (A.12) is now equivalent to

$L>\frac{2(1-\alpha)}{\omega_{p}^{2}+1}$,

where $\omega_{p}$ is determined from (A.14).
Now consider (A.8). The cross-over frequency $\omega_{c}>0$ where

$\left|\frac{1+j \alpha \omega_{c}}{1-L \omega_{c}^{2}+j(L+\alpha) \omega_{c}}\right|=1$,

can be found as the feasible root of

$L^{2} \omega_{c}^{2}\left(\omega_{c}^{2}+1-\frac{2(1-\alpha)}{L}\right)=0$.

Clearly, this has a non-zero real solution if an only if the following condition holds:

$2(1-\alpha)>L$,

in which case

$\omega_{c}=\sqrt{\frac{2(1-\alpha)}{L}-1}$.

This means that if (A.16) is not satisfied, then $|T(j \omega)|$ is a uniformly decreasing function with $T(0)=1=\|T\|_{\infty}$ which, by the small gain theorem, implies that all the roots of (A.8) are in $\mathbb{C}$. independent of $h$. Since $\omega_{p}$ is a positive real number, the condition (A.15) also holds irregardless of delay value $h$ when (A.16) is not satisfied. To complete the analysis, now assume (A.16) is satisfied and $\omega_{c}$ is as defined by (A.17). In this case, by the Nyquist criterion, all the roots of (A.8) are in $\mathbb{C}_{\text {. }}$ if and only if $\tan ^{-1}\left(\alpha \omega_{c}\right)-a \tan 2\left[(L+\alpha) \omega_{c}, 1-L \omega_{c}^{2}\right]-h \omega_{c}>-\pi$,

where $a \tan 2(y, x)=\operatorname{Pr} \arg (x+i y)=\operatorname{Arg}(x+i y)$.

To recapitulate, with the parameter definitions of (A.6), the feedback system described by (10) is stable independent of $h$ if $\alpha \geq 1$. When $\alpha<1$, system is stable independent of $h$ if $L>2(1-\alpha)>0$ and is stable depending on $h$ if $2(1-\alpha)>L>0$. For every fixed $h>0$ the region of delay-dependent stabilizing $\{(\alpha, L): 2(1-\alpha)>L>0\}$ is determined from the intersection of the conditions (A.12) and (A.18).

Since (A.17) implies

$L=\frac{2(1-\alpha)}{1+\omega_{c}^{2}}$

for $\alpha<0$ and $2(1-\alpha)>L$, the condition (A.15) can be re-written as $\omega_{p}>\omega_{c}$.

Let us now re-consider (A.18). Using the notation $L=2(1-\alpha)$ / $\left(1+\omega_{c}^{2}\right)$, then, after simple algebraic manipulations, it is easy to see that

$$
\begin{aligned}
& \tan ^{-1}\left(\alpha \omega_{c}\right)-a \tan 2\left[(L+\alpha) \omega_{c}, 1-L \omega_{c}^{2}\right] \\
& \quad=-\tan ^{-1}\left(\frac{2(1-\alpha) \omega_{c}\left(1+\alpha \omega_{c}^{2}\right)}{\left(1+\alpha \omega_{c}^{2}\right)^{2}-\left(2(1-\alpha) \omega_{c}\right)^{2}}\right) \\
& \quad=-2 \tan ^{-1}\left(\frac{(1-\alpha) \omega_{c}}{\left(1+\alpha \omega_{c}^{2}\right)}\right)=-2\left(\tan ^{-1}\left(\omega_{c}\right)-\tan ^{-1}\left(\alpha \omega_{c}\right)\right) .
\end{aligned}
$$

Thus the condition (A.18) is equivalent to

$\frac{\pi-2\left(\tan ^{-1}\left(\omega_{c}\right)-\tan ^{-1}\left(\alpha \omega_{c}\right)\right)}{\omega_{c}}>h$.

Recall from (A.19) and (A.14) that $\omega_{c}$ is restricted to satisfy $\omega_{p}>\omega_{c}$, where $\omega_{p}$ is defined from

$\frac{2\left(\pi-\left(\tan ^{-1}\left(\omega_{p}\right)-\tan ^{-1}\left(\alpha \omega_{p}\right)\right)\right)}{\omega_{p}}=h$.

Resuming, the system is stable independent of delay $h$ if $\alpha \geq 1$; or if $\alpha<1$ and $L>2(1-\alpha)$. Furthermore, the analysis for the case $\alpha<1$ and $2(1-\alpha)>L>0$ reduces to the following. Define

$g_{c}(x)=\frac{\pi-2\left(\tan ^{-1}(x)-\tan ^{-1}(\alpha x)\right)}{x}$, 
$g_{p}(x)=\frac{2\left(\pi-\left(\tan ^{-1}(x)-\tan ^{-1}(\alpha x)\right)\right)}{x}$.

Clearly, $g_{p}$ and $g_{c}$ are uniformly decreasing functions and $g_{p}(x)>g_{c}(x)$ for all $x>0$. So, if $\omega_{p}$ is defined as the solution of the equation $g_{p}(x)=h$ and $\omega_{o}$ as the solution of the equation $g_{c}(x)=h$, then $\omega_{o}<\omega_{p}$ and hence, for $\alpha<1$ and $2(1-\alpha)>L>0$, the feedback system shown in Fig. 4 is stable if and only if $\omega_{c}<\omega_{0}$, which is equivalent to:

$L>\frac{2(1-\alpha)}{1+\omega_{o}^{2}}$, where $\omega_{o}>0$ is the solution of $g_{c}(x)=h$.

\section{References}

Alfaro, V. (2007). PID controller's fragility. ISA Transactions, 46(4), 555-559.

Artigas, J., Vilanova, J., Preusche, C., \& Hirzinger, G. (2006). Time domain passivity control-based telepresence with time delay. In International conference on intelligent robots and systems (IROS) (Vol. 21, pp. 4205-4210).

Aziminejad, A., Tavakoli, M., Patel, R. V., \& Moallem, M. (2008). Stability and performance in delayed bilateral teleoperation: theory and experiments. Control Engineering Practice, 16, 1329-1343.

Çalıșkan, S. Y., Özbay, H., \& Niculescu, S.-I. (2011). Stability analysis of switched systems using Lyapunov-Krasovskii functionals. In Preprints of the 18th IFAC world congress, Milano (Italy) August 28-September 2, 2011 (pp. 7492-7496).

Cheong, J., Niculescu, S.-I., \& Kim, C. (2009). Motion synchronization control of distributed multisubsystems with invariant local natural dynamics. IEEE Transactions on Robotics, 25, 382-398.

David, O., Measson, Y., Bidard, C., Rotinat-Libersa, C., \& Russotto, F. X. (2007). Maestro: A hydraulic manipulator for maintenance and decomissionning. In European nuclear conference (ENC), Bruxelles, Belgium.

Gil, J., Sanchez, E., Hulin, T., Preusche, C., \& Hirzinger, G. (2007). Stability boundary for haptic rendering: Influence of damping and delay. In IEEE international conference on robotics and automation (pp. 124-129).

Gosselin, F., Megard, C., Bouchigny, S., Ferlay, F., Taha, F., Delcampe, P., \& D'Hauthuille, C. (2010). A VR training platform for maxillo facial surgery. In Applied human factors and ergonomics (AHFE) international conference, Miami, Florida, USA, Advances in Cognitive Ergonomics.

Hokayem, P. F., \& Spong, M. W. (2006). Bilateral teleoperation: An historical survey. Automatica, 42(12), 2035-2057.

Keel, L., \& Bhattacharyya, S. (1997). Robust, fragile or optimal? In Proceedings of the American control conference (Vol. 2, pp. 1307-1313).

Lawrence, D. A. (1993). Stability and transparency in bilateral teleoperation. IEEE Transactions on Robotics Automation, 9(5), 624-637.

Lee, D., \& Huang, K. (2008). Passive position feedback over packet-switching communication network with varying-delay and packet-loss. In Symposium on haptic interfaces for virtual environment and teleoperator systems. Haptics (pp. 335-342).

Lee, D., \& Spong, M. W. (2006). Passive bilateral teleoperation with constant time delay. IEEE Transactions on Robotics, 22(2), 269-281.

Liacu, B., Mendez-Barrios, C., Niculescu, S.-I., \& Olaru, S. (2010). Some remarks on the fragility of PD controllers for SISO systems with I/O delays. In Proceedings 14th international conference on system theory and control, Sinaia, Romania.

Liacu, B., Andriot, C., Dumur, D., Colledani, F., Niculescu, S.-I., \& Boucher, P. (2012) Experimental comparative study of control architectures for haptic interfaces including communication delays. Presented at the 20th Mediterranean conference on control and automation, Barcelone, Spain (available via IEEExplorer: 10.1109/MED.2012.6265660).

Makila, P., Keel, L., \& Bhattacharyya, S. (1998). Comments on robust, fragile or optimal? [and reply]. IEEE Transactions on Automatic Control, 43(9), 1265-1268.

Michiels, W., \& Niculescu, S.-I. (2007). Stability and stabilization of time-delay systems. An eigenvalue based approach. In Advances in design and control (Vol. 12) Philadelphia: SIAM Publications.

Morarescu, I.-C., Mendez-Barrios, C., Niculescu, S.-I., \& Gu, K. (2011). Stability crossing boundaries and fragility characterization of PID controllers for SISO systems with I/O delays. In American control conference (ACC2011), San Francisco, US.

Morarescu, I-C., Niculescu, S-I., \& Gu, K. (2010). The geometry of stability crossing curves of PI controllers for SISO systems with I/O delays. Revue Roumaine De Mathematiques Pures et Appliques, 55, 297-313.

Niemeyer, G., \& Slotine, J. J. E. (2004). Telemanipulation with time delays. The International Journal of Robotics Research, 23(9), 873-890.

Niemeyer, G. (1996). Using wave variables in time delayed force reflecting teleoperation. Ph.D. Dissertation, Massachussetts Institute of Technology.

Nuno, E., Ortega, R., Barabanov, N., \& Basanez, L. (2008). A globally stable pd controller for bilateral teleoperators. IEEE Transactions on Robotics, 24(3), 753-758.

O'Dwyer, A. (2000). PI and PID controller tuning rules for time delay process: A summary. Technical report AOD-00-01, Dublin Institute of Technology, Ireland

Rodriguez-Seda, E., Lee, D., \& Spong, M. (2009). Experimental comparison study of control architectures for bilateral teleoperators. IEEE Transactions on Robotics, 25, 1304-1318.

Ryu, J.-H., Kwon, D.-S., \& Hannaford, B. (2002a). Stable teleoperation with time domain passivity control. In IEEE international conference on robotics and automation (pp. 3260-3265). Washington, DC: ICRA.

Ryu, J.-H., Kwon, D.-S., \& Hannaford, B. (2002b). Stability guaranteed control: Time domain passivity approach. In Proceedings of the 2002 IEEE/RSJ international conference on intelligent robots and systems, Lausanne, Switzerland (Vol. 3, pp. 2115-2121).

Saeki, M. (2007). Properties of stabilizing PID gain set in parameter space. IEEE Transactions on Automatic Control, 52(9), 1710-1715.

Sankaranarayanan, G., \& Hannaford, B. (2008). Experimental comparison of internet haptic collaboration with time-delay compensation techniques. In IEEE international conference on robotics and automation (pp. 206-211). ICRA May 2008.

Shayer, L. P. \& Campbell, S. A. (2000). Stability, bifurcation and multistability in a system of two coupled neurons with multiple time delays. SIAM Journal on Applied Mathematics, 61(3), 673-700.

Sheridan, T. B. (1993). Space teleoperations through time delay: Review and prognosis. IEEE Transactions on Robotics Automation, 9, 592-606.

Silva, G. J., Datta, A., \& Bhattacharrya, S. P. (2005). PID controllers for time delays systems. Birkhauser, Boston.

Sreng, J., Lécuyer, A., Mégard, C., \& Andriot, C. (2006). Using visual cues of contact to improve interactive manipulation of virtual objects in industrial assembly/ maintenance simulations. IEEE Transactions on Visualization and Computer Graphics, 12(5), 1013-1020.

Yan, P., \& Özbay, H. (2008). Stability analysis of switched time-delay systems. SIAM Journal of Control and Optimization, 47(2), 936-949.

Yan, P., Özbay, H., \& Șansal, M. (2011). Dwell time optimization in switching control of parameter varying time delay systems. In Proceedings of the 50th IEEE conference on decision and control (pp. 4909-4914). Orlando, Florida, USA December 2011.

Yokokohji, Y., \& Yoshikawa, T. (1994). Bilateral control of master-slave manipulators for ideal kinesthetic coupling-formulation and experiment. IEEE Transactions on Robotics Automation, 10(5), 605-619. 